\title{
GA Based Estimation of Sparse MIMO Channels with Superimposed Training
}

\author{
Babar Mansoor ${ }^{1}$, Syed Junaid Nawaz ${ }^{1}$, Moazzam Islam Tiwana ${ }^{1}$, Junaid Ahmed ${ }^{1}$, Abdul Haseeb ${ }^{2}$ \\ ${ }^{I}$ Department of Electrical Engineering, COMSATS Institute of Information Technology, \\ Islamabad, 45550, Pakistan \\ ${ }^{2}$ Department of Electrical Engineering, Institute of Space Technology, \\ Islamabad, 44000, Pakistan \\ junaidnawaz@ieee.org
}

\begin{abstract}
Multiple-input multiple-output (MIMO) techniques are foreseen to play a vital role in future 5G cellular networks. This paper presents a novel approach that employs genetic algorithm (GA) to estimate the sparse uplink MIMO channels using superimposed training sequence $(\mathrm{SiT})$. At each transmitter (user) a training sequence is mathematically added with the data bits; thus, avoiding the overhead of dedicated frequency/time slots used for the training. On the receiver side (base station), signals received at all the receive antennas are jointly processed by employing the proposed method to obtain channels' estimate. Then, a linear minimum mean square error (LMMSE) equalizer estimates the data sequences sent by transmitter. A computer simulation based performance analysis of the proposed method is presented, where performance evaluation is done using metrics of normalized channel mean square error (NCMSE), as well as, bit error rate (BER). A comparative analysis of the proposed method with notable SiT least squares (SiT-LS) and SiT-LMMSE methods in the literature is conducted, which clearly demonstrates that the proposed method outperforms both the existing techniques.
\end{abstract}

Index Terms-Channel estimation; Equalization; MIMO; Genetic algorithms; Superimposed training.

\section{INTRODUCTION}

The wide spread usage of smart phones with data hungry applications has resulted in an enormous growth in the data traffic. It is foreseen that the data traffic in cellular communication networks will grow up to 500 -folds by the year 2020 [1]. The scarcity of the available electromagnetic spectrum is a major impediment in meeting the demands for higher data rates, thus, posing serious challenges to the modern cellular networks. In order to meet these challenges, research community is exploring new solutions in terms of technologies and architectures for the future cellular communication networks. A vital solution to meet these challenges is to use the substantial millimetre wave (mmWave) spectrum, which is unoccupied and underutilized to a large extent [2]. Another prominent solution is the spatial reuse of the available electromagnetic spectrum by employing multiple transmit/receive antennas. Such multiple-input multiple-output (MIMO) systems have been incorporated in the modern cellular networks such as

Manuscript received 26 March, 2018; accepted 4 August, 2018.

The authors would like to acknowledge the partial support by EU funded research project ATOM-690750 (H2020-MSCA-RISE-2015).
LTE-Advanced. Massive MIMO, which employs a large number of antennas at the base station (BS), is a prospective technology for emerging cellular networks due to its magnificent gains in spectral and energy efficiency as compared to conventional MIMO systems [3], [4]. However, the acquisition of an accurate estimate of the channel state information (CSI) is of vital importance for harvesting the substantial advantages of Massive MIMO.

Various empirical studies in the existing literature have established the fact that certain propagation environments lead to a channel impulse response (CIR) that exhibits a sparse structure in spatial, angular or delay domain. A sparse CIR is characterized by few dominant channel taps relative to the length of the channel. A sparse structured CIR is observed in communication environments such as aeronautical communications [5], underwater acoustic communications [6], and wideband high frequency communications [7]. It is established in [8] that MIMO communication channels tend to exhibit a joint sparsity and possess a common support. It is demonstrated in [9] that mmWave based communication systems also tend to have a sparse structured CIR. A prior available knowledge about the sparse nature of a communications channel can be effectively utilized for obtaining the estimate of CSI.

In the existing literature, several blind, semiblind and training based channel estimation techniques have been proposed for MIMO communication systems - see e.g., [10]-[12]. However, recently channel estimation techniques which are based on the approach of superimposed training (SiT) have gained significant attention due to their certain advantages over the counterparts [13]-[16]. SiT based channel estimation techniques achieve enhanced spectral efficiency by evading the need for dedicated time/frequency slots allocation to the training sequence. In SiT based channel estimation techniques, a low power and periodic training sequence is mathematically added over the information sequence at the transmitter side. The periodic structure of the training sequence is exploited by the receiver to estimate CSI. A user sum-rate based comparison of time multiplexed and superimposed arrangement of pilots is conducted in a multi-cell scenario in [17], where it is demonstrated that SiT based pilot arrangement schemes are superior with inherent capability of mitigating pilot contamination in large-scale MIMO systems. 
Genetic algorithms (GA) based optimization methods use stochastic search algorithms to efficiently reach an optimized solution which is in a big solution space. GAs model nature's biological evolution, crossover and mutation processes to achieve a complex optimization objective function. When sufficient number of chromosomes are used, the GA has the advantage of not getting stuck in local minimas. GA based optimization algorithms are used in a variety of fields including channel estimation in wireless communications [20]. This paper utilises GA for channel estimation using SiT technique for a MIMO sparse multipath channels, which, to our knowledge, has not been done before. The main contributions of this paper are stated as under:

1. An SiT based estimation technique is proposed for the frequency-selective sparse MIMO channels.

2. GAs are exploited for the purpose of channel estimation and the impact of variations in population size of GAs on the performance of channel estimation is studied.

3. Impact of variations in channels' sparsity level and training to information power ratio on normalized channel mean square error (NCMSE) is thoroughly studied.

4. A comprehensive comparison of the proposed technique with the existing least squares (LS) and linear minimum mean square error (LMMSE) estimation techniques is conducted.

The rest of the paper is organized as follows: the multiuser MIMO communication system model is presented in Section II. The details of the proposed channel estimation technique, for sparse MIMO channels that utilizes GA, are presented in Section III. Section IV explains the LMMSE equalizer incorporated in the system model. Section V describes the performance analysis using the performed simulations. Section VI presents the conclusion of the paper.

\section{SYSTEM MODEL}

The details of the proposed system model are illustrated in Fig. 1 such that $N$ denotes the mobile user terminals and $M$ denotes the elements in receiving antenna array. The signals as transmitted by $M$ users travels through the sparse MIMO channel. $L$ denotes the number of resolvable multipaths in such channel, out of which $Q$ are non-zero paths. The Section III details the channel estimator (CE) block that uses the first order statistics [13], as well as GA based superimposed training sequence estimation. Once an estimate of the CIR is obtained, the training sequence's contribution is removed from the resulting signal using training effect remover (TER) and then it is fed to equalizer. Subsequently, the transmitted information sequence is estimated using LMMSE equalizer. The transmitted information sequence for $n^{\text {th }}$ user, denoted by $b_{n}$, is zero mean and mutually independent for each of the $n$ users. The vector form of the information sequence is $\boldsymbol{b}_{n}=\left[b_{n}(0), b_{n}(1), \ldots, b_{n}(k-1)\right]^{*}, \quad$ while the known periodic training sequence is $\boldsymbol{c}_{n}=\left[c_{n}(0), c_{n}(1), \ldots, c_{n}(k-1)\right]^{*}$. The period of training sequence is denoted by $P$, such that $c_{n}(k)=c_{n}(k+a P)$, for integers $a$ and $P$. The information sequence with superimposed training sequence, denoted $\boldsymbol{x}_{n}$, is given as

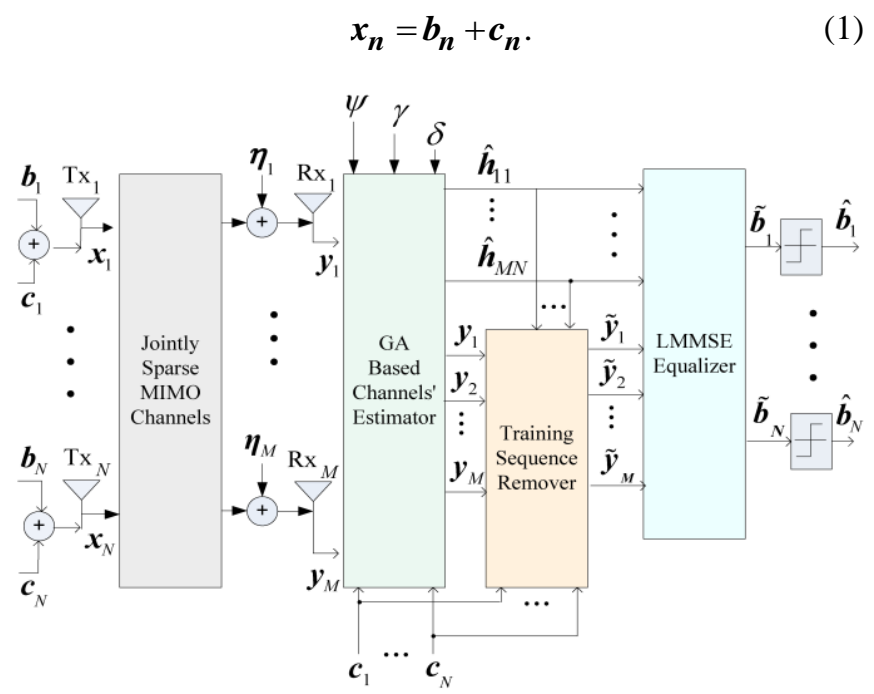

Fig. 1. Block diagram of the proposed system model for multi-user MIMO communication systems.

The transmitted signals $x_{n}$ propagate through the sparse frequency-selective MIMO channels. After reception by $m^{\text {th }}$ antenna element, the CIR between user $n$ and antenna $m$, can be denoted as $\boldsymbol{h}_{n m}=\left[h_{n m}^{0}, h_{n m}^{1}, . ., h_{n m}^{L-1}\right]^{*}$. At time instant $k$, the signal received at antenna $m$, is expressed as

$$
y_{m}(k)=\sum_{n=1}^{N} \sum_{l=0}^{L-1} h_{n m}^{l} x_{n}(k-l)+\eta_{m}(k),
$$

where $\eta_{m}(k)$ represents additive white Gaussian noise (AWGN) on antenna $m$ and at time instant $k$, with a variance of $\sigma_{\eta}^{2}$. The overall instantaneous signal as captured by $\mathrm{m}^{\text {th }}$ antenna array element at instant $k$ is $\boldsymbol{y}(k)=\left[y_{1}(k), y_{2}(k), \ldots, y_{M}(k)\right]^{*}$, and is calculated as

$$
\boldsymbol{y}(k)=\sum_{l=0}^{L-1} \mathbf{H}^{l} \boldsymbol{x}(k-1)+\boldsymbol{\eta}(k),
$$

where $\mathbf{H}^{l}$ is the channel matrix for $l^{\text {th }}$ delay tap with dimensions $M \times N$

$$
\mathbf{H}^{l}=\left[\begin{array}{cccc}
h_{11}^{l} & h_{12}^{l} & \ldots & h_{1 M}^{l} \\
h_{21}^{l} & h_{22}^{l} & \ddots & h_{2 M}^{l} \\
\vdots & \vdots & \ddots & \vdots \\
h_{N 1}^{l} & h_{N 2}^{l} & \ldots & h_{N M}^{l}
\end{array}\right\rfloor
$$

while the parameters $\boldsymbol{\eta}(k)=\left[\eta_{1}(k), \eta_{2}(k), \ldots, \eta_{M}(k)\right]^{*}$, and $\boldsymbol{x}(k-l)=\left[x_{1}(k-l), x_{2}(k-l), \ldots, x_{n}(k-l)\right]^{*}$. After temporal sampling of the signal, vector representation is given by

$$
\breve{\boldsymbol{y}}(k)=\left[\boldsymbol{y}^{*}(k+L-1), \boldsymbol{y}^{*}(k+L-2), \ldots, \boldsymbol{y}^{*}(k)\right]^{*},
$$




$$
\breve{\boldsymbol{y}}(k)=\mathbf{H} \breve{\boldsymbol{x}}(k)+\breve{\boldsymbol{\eta}}(k)
$$

where $\breve{\boldsymbol{x}}(k)$ and $\breve{\boldsymbol{\eta}}(k)$ can be represented as

$$
\begin{aligned}
& \breve{\boldsymbol{x}}(k)=\left[\boldsymbol{x}^{*}(k+L-1), \boldsymbol{x}^{*}(k+L-2), \ldots, \boldsymbol{x}^{*}(k)\right]^{*}, \\
& \breve{\boldsymbol{\eta}}(k)=\left[\boldsymbol{\eta}^{*}(k+L-1), \boldsymbol{\eta}^{*}(k+L-2), \ldots, \boldsymbol{\eta}^{*}(k)\right]^{*} .
\end{aligned}
$$

The convolution matrix $\mathbf{H}$ of MIMO channels can be represented as

$$
\mathbf{H}=\left[\begin{array}{ccccc}
\mathbf{H}^{0} & \mathbf{H}^{1} & \ldots & \mathbf{H}^{L-1} & 0 \\
\vdots & \ddots & \ddots & \ddots & \vdots \\
0 & \mathbf{H}^{0} & \mathbf{H}^{1} & \ldots & \mathbf{H}^{L-1}
\end{array}\right\rfloor
$$

\section{PROPOSED GA BASED METHOD FOR SPARSE MIMO CHANNEL ESTIMATION}

An overview of received signals' first-order statistics, exploited in the proposed work, is discussed in Section III-A and Section III-B. The conventional least squares based channel estimation is discussed in Section III-C while our proposed GA based channel estimation is detailed in the Section III-D.

\section{A. Superimposed Training Sequence Design}

A distinct cyclic frequency had been assigned to each mobile user. Let $c_{n}(k)$ be the training sequence of the $n^{\text {th }}$ mobile user. $P=\tilde{P} N$ denotes the training sequence period, such that $\tilde{P} \in Z^{+}$. The training sequence $c_{n}(k)$ can be expressed as

$$
c_{n}(k)=\sum_{i=0}^{P-1} c_{i, n} e^{j(2 \pi i / P) k}
$$

where $\forall k, j=\sqrt{-1}$. Furthermore

$$
c_{i, n}=\frac{1}{P} \sum_{k=0}^{P-1} c_{n}(k) e^{j(2 \pi i / P) k}
$$

The training sequence $c_{n}(k)$ has been chosen so that out of $P$ coefficients, $\tilde{P}$ are not zero. Calculate $c_{n}(k)$ as expressed below

$$
c_{n}(k)=\sum_{i=0}^{\tilde{P}-1} c_{i, n}^{\prime} e^{j \alpha_{i, n} k}
$$

where $\forall k, \alpha_{i, n}=2 \pi(i N+n-1) / P$. The coefficients $c_{i, n}^{\prime}$ are suitably chosen for $1 \leq n \leq N$ and $0 \leq i \leq \tilde{P}-1$. For the calculation of $c_{n}(k)$ a base sequence $\bar{c}_{0}(k)$ is needed. $\tilde{P}$ is the period of $c_{o}(k)$, such that

$$
\bar{c}_{i, o}=\frac{1}{\tilde{P}} \sum_{k=0}^{\tilde{P}-1} \bar{c}_{0}(k) e^{j(2 \pi i / \tilde{P}) k}
$$

The training sequence $\bar{c}_{1}(k)$ with a period $P$, can be defined by repeating $\bar{c}_{0}(k) N$ times. Now, $c_{n}(k)$ for the $n^{\text {th }}$ user can be calculated as

$$
c_{n}(k)=\sigma_{c_{n}} \bar{c}_{1}(k) e^{j(2 \pi / P)(n-1) k} .
$$

\section{B. First-Order Statistics of the Received Signal}

The signal received at $k^{\text {th }}$ time instant and $m^{\text {th }}$ element of receiver is represented by $y_{m}(k)$, such that its statistical expectation is given as

$$
E\left\{y_{m}(k)\right\}=\sum_{n=1}^{N} \sum_{i=0}^{\tilde{P}-1}\left[\sum_{l=0}^{L-1} c_{i, n}^{\prime} h_{n m}^{l} e^{-j \alpha_{i, n} l}\right] e^{j \alpha_{i, n} k}
$$

where $\quad n_{1} \neq n_{2}, \quad$ and $\quad \alpha_{i_{1}, n_{1}} \neq \alpha_{i_{2}, n_{2}} . \quad$ such that $\left\{i_{1}, i_{2}\right\} \in 0,1, \ldots, P-1$. Defining the vector $\boldsymbol{d}_{n m}$ as, $\boldsymbol{d}_{n m}=\left[d_{n m, 0}, d_{n m, 1}, \ldots, d_{n m,(\tilde{P}-1)}\right]^{*}$, such that $d_{n m, i}$ can be represented by

$$
d_{n m, i}=\sum_{l=0}^{L-1} c_{i, n}^{\prime} h_{n m}^{l} e^{-j \alpha_{i, n} l}
$$

Let $\hat{\boldsymbol{d}}_{n m}=\left[\hat{d}_{n m, 0}, \hat{d}_{n m, 1}, \ldots, \hat{d}_{n m,(\tilde{P}-1)}\right]^{*}$ be the meansquare consistent estimate of $\boldsymbol{d}_{n m}$. To calculate the estimate $\hat{d}_{n m}$, the coefficients can be computed as

$$
\hat{d}_{n m, i}=\frac{1}{\breve{K}} \sum_{k=0}^{\breve{K}-1} y_{m}(k) e^{-j \alpha_{i, n} k}
$$

where $\breve{K}$ represents the total number of symbols received. For $\breve{K} \rightarrow \infty$, consequently $\hat{d}_{n m, i} \rightarrow d_{n m, i}$. Let $\varepsilon_{n m, i}$, denote the error between $d_{n m, i}$ and $\hat{d}_{n m, i}$. Hence, we can write

$$
\hat{d}_{n m, i}=d_{n m, i}+\varepsilon_{n m, i}
$$

The error in estimation $\varepsilon_{n m, i}$ is a result of cumulative effect of additive noise, interference experienced due to superimposed information sequences transmitted by other users, as well as superimposed training sequences used on other channels. Writing (17) as vectors yields

$$
\hat{\boldsymbol{d}}_{n m}=\mathbf{C}_{n} \boldsymbol{h}_{n m}
$$

where $\mathbf{C}_{\mathrm{n}}$ is given as follows 


$$
\mathbf{C}_{n}=\operatorname{diag}\left\{c_{0, n}^{\prime}, c_{1, n}^{\prime}, \ldots, c_{(\tilde{P}-1), n}^{\prime}\right\} \times \mathbf{V}_{n},
$$

where $\mathbf{V}_{n}$ can be calculated as

$$
\mathbf{V}_{n}=\left[\begin{array}{cccc}
1 & 1 & \cdots & 1 \\
1 & e^{-j \alpha_{1, n}} & \cdots & e^{-j \alpha_{1, n} L} \\
\vdots & \vdots & \ddots & \vdots \\
1 & e^{-j \alpha_{(\beta-1), n}} & \cdots & e^{-j \alpha_{(\beta-1), n} L}
\end{array}\right]
$$

\section{Least Squares and LMMSE Based Channel Estimation}

Use (19) to calculate channel's least square estimate [20], as follows

$$
\hat{\boldsymbol{h}}_{n m}^{\mathrm{SiT}-\mathrm{LS}}=\left(\mathbf{C}_{n}^{*} \mathbf{C}_{n}\right)^{-1} \mathbf{C}_{n}^{*} \hat{\boldsymbol{d}}_{n m}
$$

In case of noise with non-zero mean, the channel can be calculated using the condition $\tilde{P} \geq L+1$, and removing $\hat{d}_{n m, 0}$ from $\hat{\boldsymbol{d}}_{n m}$ and removing first row of $\mathbf{C}_{n}$. An LMMSE based estimate of the sparse CIR vectors is given by

$$
\begin{aligned}
\hat{\boldsymbol{h}}_{n m}^{\text {SiT-LMMSE }} & =\mu_{h}+\left(\mathbf{C}_{n}^{*} \mathbf{C}_{n}+\sigma_{\eta}^{2} \boldsymbol{R}_{H H}^{-1}\right)^{-1} \times \\
& \times \mathbf{C}_{n}^{*}\left(\hat{\boldsymbol{d}}_{n m}-\mu_{d}\right),
\end{aligned}
$$

where $\mathbf{R}_{H H}$ is the channel covariance matrix and is assumed to be known at the receiver, $\mu_{d}$ is the mean of $\hat{\boldsymbol{d}}_{n m}$, and $\mu_{h}$ is the mean of the channel coefficients.

\section{Proposed GA Based Channel Estimation}

GAs are evolutionary search based techniques that have been frequently utilized to solve channel equalization and estimation problems in challenging wireless environments. This section presents a novel GA assisted algorithm that has been used for sparse multipath MIMO channels' estimation. It uses the first order statistics calculated from the received signal as well as the a priori information about channel's sparsity. The estimation error $\boldsymbol{\xi}$ of the cost function is reduced using the compensation factor $\epsilon$. GAs use stochastic search to find the near optimal solution in the entire solution space while at the same time minimizing the objective function given as:

$$
\begin{gathered}
\widetilde{\boldsymbol{h}}_{n m}=\underset{\tilde{\boldsymbol{h}}_{\boldsymbol{n} \boldsymbol{m}}}{\arg \min }\left\|\tilde{\boldsymbol{h}}_{n m}\right\|_{\ell 1}, \text { subject to, } \\
\left\{\begin{array}{l}
\left\|\boldsymbol{C}_{\boldsymbol{n}} \tilde{\boldsymbol{h}}_{\boldsymbol{n} \boldsymbol{m}}-\tilde{\boldsymbol{d}}_{\boldsymbol{n} \boldsymbol{m}}\right\|_{\ell 2}^{2} \leq \tilde{\varepsilon} \epsilon, \\
\left\|\tilde{\boldsymbol{h}}_{\boldsymbol{n} \boldsymbol{m}}\right\|_{\ell 2} \leq 1,
\end{array}\right.
\end{gathered}
$$

where $\epsilon \tilde{\varepsilon} \propto\|\xi\|_{\ell 2}^{2}$. Owing to sparsity of the channel, an $\ell 1-$ norm based objective function has been considered for minimization [21]. GA has a population size of $\gamma$ individual chromosomes where each chromosome corresponds to a solution vector in the solution space. At the start of GA, initial chromosome population $\tilde{\boldsymbol{h}}_{n m, 0}$ corresponds to $0^{\text {th }}$ generation (i.e., $g_{0}$ ), and can be generated randomly. An intelligent guess for the initial population $\tilde{\boldsymbol{h}}_{n m, 0}$ can be the SiT-LS estimate obtained in (1). The genetic algorithms are iterative in nature. The genetic algorithms successively employ the selection, crossover, and mutation operators to derive the population $\tilde{\boldsymbol{h}}_{n m, i}$ of $g_{n m, i}$ ( $i^{\text {th }}$ generation) from population $\tilde{\boldsymbol{h}}_{n m, i-1}$ of previous $g_{n m, i-1}$. The chromosomes in $\tilde{\boldsymbol{h}}_{n m, i-1}$ are evaluated sequentially one-by-one by using the fitness/objective function. Based upon this, evaluation chromosomes are sorted. Then stochastic uniform sampling method in selection process is used to select the better performing chromosomes, called parent chromosomes. Among these selected chromosomes, those having the lowest score pass without any change, as children chromosome to $\tilde{\boldsymbol{h}}_{n m, i}$. While operators of crossover or mutation are employed on remaining parent chromosomes to generate the remaining children chromosomes in $\tilde{\boldsymbol{h}}_{n m, i}$ The crossover operation involves randomly combining two parent chromosomes to generate a child chromosome for $g_{n m, i}$. While in mutation, an individual parent chromosome undergoes changes or mutation to generate a child chromosome mutation avoid the possibility of GA getting stuck in local minima. Hence, the possibility of prematurely converging to a suboptimal solution is reduced. Then, $\tilde{\boldsymbol{h}}_{n m, i}$ gets replaced by $\tilde{\boldsymbol{h}}_{n m, i-1}$. These steps are repeated iteratively to minimize the defined objective function, until a pre-set convergence criterion or the maximum number of generations $(\psi)$ are met. The pre-set convergence criteria can be defined as when the fitness values of objective function for two successive generations becomes less than $\delta$. Finally, the solution corresponding to the chromosome with least value of fitness gives $\breve{\boldsymbol{h}}_{n m}$, i.e., the required sparse multipath channel's estimate.

\section{LMMSE EQUALIZER WITH THE PROPOSED TECHNIQUE}

The receiver knows the superimposed training sequence transmitted by each mobile user. At the receiver, CIR is convolved with the superimposed training sequence. So, before estimation of information sequence, the contamination caused by training sequence in the information sequence needs to be normalized. This elimination of training sequences' effect at each receiver element is performed by training sequence effect remover block (Fig. 1), which can be expressed as follows

$$
\tilde{y}_{m}(k)=y_{m}(k)-\sum_{n=1}^{N} \sum_{l=0}^{L-1} \breve{h}_{n m}^{l} c_{n}(k-l),
$$

where $\breve{h}_{n m}^{l}$ denotes the channel's $l^{\text {th }}$ tap, as seen from $n^{\text {th }}$ mobile user towards the receiver's $m^{\text {th }}$ antenna element. The GA based channel estimation technique has been detailed in the previous section. After removing training sequence's effect, the resulting signal is then provided to the equalizer 
for computing the estimate of information sequence. Let $\boldsymbol{w}_{n}$ be the optimal equalizer weight corresponding to the $m^{\text {th }}$ antenna element, and is estimated as [22]: subtraction

$$
\boldsymbol{w}_{n}=\left.\left(\breve{\mathbf{H}} \breve{\mathbf{H}}^{*}+2 \hat{\sigma}_{n}^{2} \mathbf{I}\right)^{-1} \breve{\mathbf{H}}\right|_{(m-1)\left(L_{e}+L-1\right)+(\tau+1)},
$$

where $L_{e}$ represents the number of equalizer taps, $\mathbf{I}$ is the identity matrix with dimensions $L_{e} \times L_{e}, \tau$ denotes the mapping delay, and $\left.\breve{\mathbf{H}}\right|_{i}$ represents the $i^{\text {th }}$ column of $\breve{\mathbf{H}}$.

While $\breve{\mathbf{H}}$ denotes the convolutional matrix of dimensions $L_{e} \times\left(L_{e}+L-1\right)$. The estimated impulse response vectors of channels can be used to calculate $\breve{\mathbf{H}}_{n m}$ as follows

$$
\begin{gathered}
\breve{\mathbf{H}}_{n m}= \\
=\left[\begin{array}{cccccccc}
\breve{h}_{n m}^{0} & \breve{h}_{n m}^{1} & \ldots & \breve{h}_{n m}^{L} & 0 & 0 & \ldots & 0 \\
0 & \breve{h}_{n m}^{0} & \breve{h}_{n m}^{1} & \ldots & \breve{h}_{n m}^{L} & 0 & \ddots & 0 \\
\vdots & \ddots & \ddots & \ddots & \ddots & \ddots & \ddots & \vdots \\
0 & 0 & \ldots & 0 & \breve{h}_{n m}^{0} & \breve{h}_{n m}^{1} & \ldots & \breve{h}_{n m}^{L}
\end{array}\right] .
\end{gathered}
$$

Subsequently, we can calculate $\breve{\mathbf{H}}$, the composite representation of MIMO channels' convolutional matrices, as

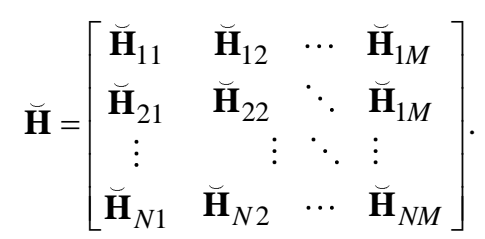

The estimate of additive noise variance $\hat{\sigma}_{n}^{2}$ can be obtained as given in [13]. Finally, the transmitted sequence of $n^{\text {th }}$ transmitter is calculated as

$$
\tilde{b}_{n}(k)=\sum_{m=1}^{M} \sum_{i=0}^{L_{e}-1} w_{n m, i} \tilde{y}_{m}(k-i)
$$

where $\tilde{b}_{n}$ is the equalizer output, and it serves as the input of decision mapper, as explained in Fig. 1. The decision mapper then calculates the decoded symbols $\hat{b}_{n}$, by mapping symbols in accordance with the selected modulation schemes.

\section{Simulation Results}

This section conducts the performance analysis of SiTGA method based upon the results of performed computer simulations. In the simulation setup, the realization of channels $\boldsymbol{h}_{n m}$ are generated independently for each Monte Carlo run, while the channels' sparsity level is kept fixed at $Q / L$.

The positions of non-zero values in the CIR vector are drawn from a uniform distribution. While a zero-man Gaussian distribution with $1 / M(L+1)$ variance, is used to draw the values of non-zero channel taps. The channels from a particular user terminal to all the receive antenna elements are assumed to possess a common support (nonzero tap positions) because of the small antenna separations at the receiver. Binary phase shift keying (BPSK) modulated data symbols are assumed to be uniformly distributed with zero mean and variance $\sigma_{b}^{2}$, and are generated independently for each user (transmitter) and for each Monte Carlo run. At each receive antennas element, the signal-to-noise ratio (SNR) is specified as the ratio of power of received signal to the power of AWGN; where it is assumed that received SNR at each antenna is equal. The impact of population size on the MSE and BER performance of proposed method can be seen in Fig. 2(a) and Fig. 2(b), respectively. It is evident that increasing the population size results in an improvement in both MSE and BER performance; however, the rate of increase in marginal for population size higher than $4 L$. The effect of channels' sparsity level on MSE performance is plotted in Fig. 3(a). The performance of proposed method is observed to improve with an increase in channels' sparsity. The impact of increase in training to information power ratio in MSE performance of the proposed method is plotted in Fig. 3(b). An increase in power of the training sequence compared to the information sequence results in an improvement in the accuracy of channel estimate calculated at the receiver. However, relative decrease in the power of information sequence beyond $\sigma_{c}^{2} / \sigma_{b}^{2}=0.6$ leads to a decrease in BER performance of the system. The usefulness of proposed method is demonstrated through the comparison of the proposed SiT-GA method with notable channel estimation methods named as SiT-LS and SiT-LMMSE. The NCMSE and BER are the performance metrics used for this comparison in Fig. 4. For an SNR of $10 \mathrm{~dB}$ and channels sparsity level of $Q / L=6 / 14$, an improvement of $7.93 \mathrm{~dB}$ and $5 \mathrm{~dB}$ in MSE performance by the proposed SiT-GA method compared to SiT-LS and SiT-LMMSE, respectively, as can be observed in Fig. 4(a).

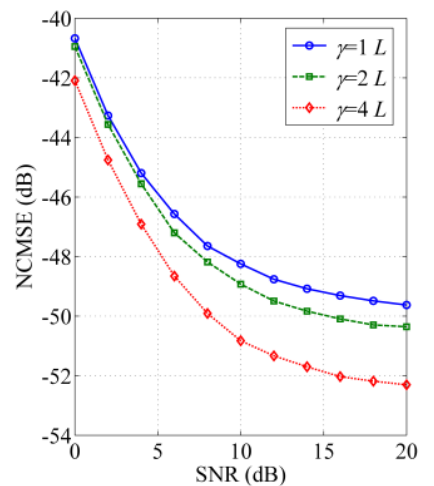

(a)

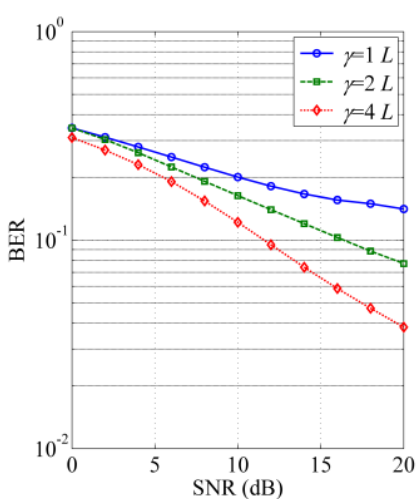

(b)
Fig. 2. The impact of population size and SNR on the NCMSE performance (a); The impact of population size and SNR on BER performance $\left(\widetilde{K}=10^{3}, \quad N=4, \quad \tau=0, \quad Q / L=4 / 14, \quad \delta=0.001\right.$, $L_{e}=15, \psi=300$, Monte $=10^{3}$ runs ) (b).

For a BER of $10^{-1}$ and channels' sparsity level of $Q / L=6 / 14$, the proposed method provides an approximate SNR gain of $3 \mathrm{~dB}$ and $2 \mathrm{~dB}$ as compared to 
SiT-LS and SiT-LMMSE methods.

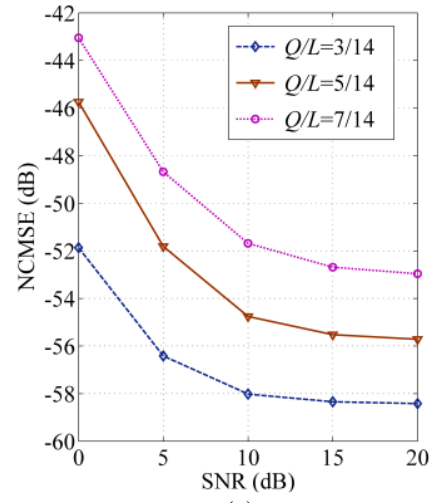

(a)

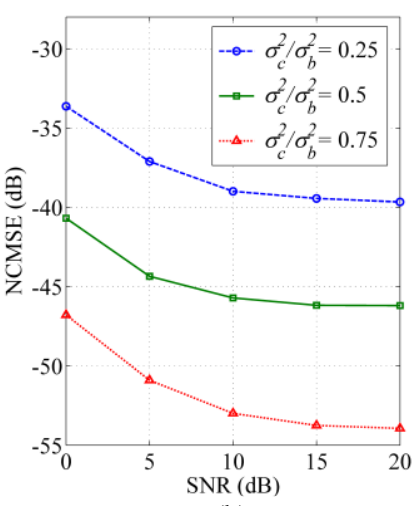

(b)
Fig. 3. The effect of channel sparsity level and SNR on NCMSE performance, for $\sigma_{c}^{2} / \sigma_{b}^{2}=0.2$ (a); the effect of training-to-information power ratio on NCMSE performance, for $Q / L=4 / 14$. $(M=4$, $N=4, \gamma=5 L, \psi=300, \delta=0.001$, and Monte $=10^{3}$ runs ) (b).

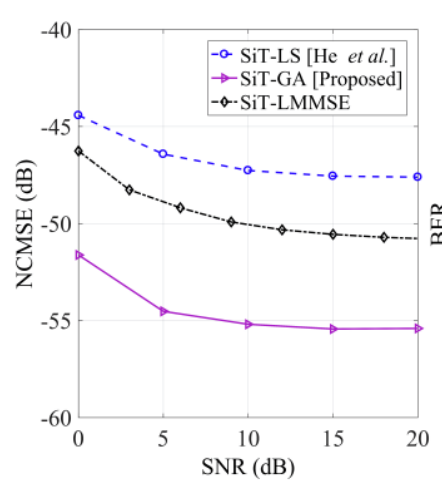

(a)

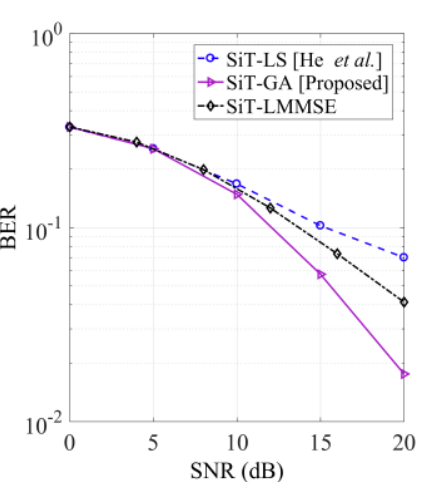

(b)
Fig. 4. NCMSE based performance comparison of the proposed method with SiT-LS [13] and SiT-LMMSE (a); BER based performance comparison of the proposed method with SiT-LS [13] and SiT-LMMSE. $\left(\breve{K}=10^{3}, M=3, N=3, Q / L=6 / 14, \psi=300, \gamma=3 L\right.$, $\sigma_{c}^{2} / \sigma_{b}^{2}=0.25, \delta=0.001, L_{e}=15$, and Monte $=10^{3}$ runs ) (b).

It is also evident that the gain offered by the proposed method in MSE and BER performance, further increases for high degree of channel sparsity. It is thus established that the proposed method promises an improved quality of service (QoS) by offering significant improvement in accuracy of channels' estimate and retrieved information.

\section{CONCLUSIONS}

A GA based method for estimation of sparse MIMO channels using superimposed training sequence has been proposed. It has been established that for a channels' sparsity level of $Q / L=6 / 14$ and training-to-information power ratio of $\sigma_{c}^{2} / \sigma_{b}^{2}=0.25$, the proposed channel estimation method outperforms the existing SiT-LS and SiTLMMSE techniques with a gain of about $7.93 \mathrm{~dB}$ and $5 \mathrm{~dB}$ in NCMSE at an SNR of $10 \mathrm{~dB}$. Similarly, for a BER of $10^{-1}$, a performance gain of $3 \mathrm{~dB}$ and $2 \mathrm{~dB}$ in SNR has been observed over the existing SiT-LS and SiT-LMMSE techniques, respectively. Moreover, it has been also established that the increased sparsity level of the MIMO channels leads to a further improvement in NCMSE of the proposed technique. Furthermore, it is observed that increasing the training-to-information power ratio results in an improvement in the channel estimate. However, allocating more power to the training sequence causes reduction in SINR at the receiver. Therefore, an optimal value of the training power needs to be chosen.

\section{ACKNOWLEDGMENT}

The authors would like to acknowledge the travel grant support by Higher Education Commission (HEC) Pakistan to attend the conference.

\section{REFERENCES}

[1] T. N. Nan, S. Nagata, A. Benjebbour, Y. Kishiyama, T. Hai, S. Xiaodong, Y. Ning, L. Nan, "Trends in small cell enhancements in LTE advanced", IEEE Commun. Mag., vol. 51, no. 2, p. 98-105, 2013. DOI: 10.1109/MCOM.2013.6461192.

[2] R. Haines, W. H. Chin, Z. Fan, "Emerging technologies and research challenges for 5G wireless networks", IEEE Wireless Commun. vol. 21, no. 2, pp. 106-112, 2014. DOI: 10.1109/ MWC.2014.6812298.

[3] F. Rusek, D. Persson, B. K. Lau, E. G. Larsson, T. L. Marzetta, O. Edfors, F. Tufvesson, "Scaling up MIMO: Opportunities and challenges with very large arrays", IEEE Signal Process. Mag., vol. 30, no. 1, pp. 40-60, 2013. DOI: 10.1109/MSP.2011.2178495.

[4] E. Larsson, O. Edfors, F. Tufvesson, T. Marzetta, "Massive MIMO for next generation wireless systems", IEEE Commun. Mag., vol. 52, no. 2, pp. 186-195, 2014. DOI: 10.1109/MCOM .2014.6736761.

[5] F. K. Lee, P. J. McLane, "Design of nonuniformly spaced tapped delay-line equalizers for sparse multipath channels", IEEE Trans. on Commun., vol. 52, pp. 530-535, 2004. DOI: 10.1109/TCOMM. 2004.826351 .

[6] Z. Jun-yi, W.-x. Meng, J. Shi-lou, "Sparse underwater acoustic OFDM channel estimation based on superimposed training", $J$. of Marine Science and Application, vol. 8, no. 1, pp. 65-70, 2009. DOI: 10.1007/s11804-009-8015-2.

[7] J. Ying, J. Zhong, M. Zhao, Y. Cai, "Turbo equalization based on compressive sensing channel estimation in wideband HF systems", in Int. Conf. on Wireless Commun. Signal Process., 2013. DOI: 10.1109/WCSP.2013.6677272.

[8] Y. Barbotin, A. Hormati, S. Rangan, M. Vetterli, "Estimation of sparse MIMO channels with common support", IEEE Trans. on Commun., vol. 60, no. 12, pp. 3705-3716, 2012. DOI: 10.1109/TCOMM.2012.091112.110439.

[9] A. Alkhateeb, O. E. Ayach, G. Leus, R. W. Heath, "Channel estimation and hybrid precoding for millimeter wave cellular systems", IEEE J. of Sel. Topics in Signal Process., vol. 8, no. 5, pp. 831-846, 2014. DOI: 10.1109/JSTSP.2014.2334278.

[10] S. Shahbazpanahi, A. B. Gershman, J. H. Manton, "Closed-form blind MIMO channel estimation for orthogonal space-time block codes", IEEE Trans. on Signal Process., vol. 53, no. 12, pp. 4506-4517, 2005. DOI: 10.1109/TSP.2005.859331.

[11] Y. Zeng, A. R. Leyman, T. S. Ng, "Joint semiblind frequency offset and channel estimation for multiuser MIMO-OFDM uplink", IEEE Trans. on Commun., vol. 55, no. 12, pp. 2270-2278, 2005. DOI: 10.1109/TCOMM.2007.910637.

[12] C. Fragouli, N. Al-Dhahir, W. Turin, "Training-based channel estimation for multiple-antenna broadband transmissions", IEEE Trans. on Wireless Commun., vol. 2, no. 2, pp. 384-391, 2003. DOI: 10.1109/TWC.2003.809454.

[13] S. He, J. K. Tugnait, X. Meng, "On superimposed training for MIMO channel estimation and symbol detection", IEEE Trans. on Signal Process., vol. 55, no. 6, pp. 3007-3021, 2007. DOI: 10.1109/TSP.2007.893941.

[14] S. J. Nawaz, K. I. Ahmed, M. N. Patwary, N. M. Khan, "Superimposed training-based compressed sensing of sparse multipath channels", IET Commun., vol. 18, no. 6, pp. 3150-3156, 2012. DOI: 10.1049/iet-com.2012.0162.

[15] B. Mansoor, S. J. Nawaz, B. Amin, S. K. Sharma, M. N. Patwary, "Superimposed training based estimation of sparse MIMO channels for emerging wireless networks", in 23rd Int. Conf. on Telecommun. (ICT 2016), 2016. DOI: 10.1109/ICT.2016.7500477.

[16] B. Mansoor, S. J. Nawaz, S. M. Gulfam, "Massive-MIMO sparse uplink channel estimation using implicit training and compressed sensing", Applied Sciences, vol. 7, no. 1, 2017. DOI: 10.3390/app7010063. 
[17] K. Upadhya, S. A. Vorobyov, M. Vehkapera, "Superimposed pilots are superior for mitigating pilot contamination in massive MIMO", IEEE Trans. on Signal Process., vol. 65, no. 11, pp. 2917-2932, 2017. DOI: $10.1109 /$ TSP.2017.2675859.

[18] K. Yen, L. Hanzo, "Genetic algorithm assisted joint multiuser symbol detection and fading channel estimation for synchronous CDMA systems", IEEE J. on Sel. Areas in Commun., vol. 19, no. 6, pp. 985998, 2001. DOI: 10.1109/49.926355.

[19] M. Jiang, J. Akhtman, L. Hanzo, "Iterative joint channel estimation and multi-user detection for multiple-antenna aided OFDM systems", IEEE Trans. on Wireless Commun., vol. 6, no. 8, pp. 2904-2914, 2007. DOI: 10.1109/TWC.2007.05817.
[20] E. J. Candes, T. Tao, "Decoding by linear programming", IEEE Trans. Inf. Tech., vol. 51, no. 12, pp. 4203-4215, 2005. DOI: 10.1109/TIT.2005.858979.

[21] W. U. Bajwa, J. Haupt, A. M. Sayeed, R. Nowak, "Compressed channel sensing: a new approach to estimating sparse multipath channels", in Proc. IEEE, vol. 98, no. 6, pp. 1058-1076, 2010. DOI: 10.1109/JPROC.2010.2042415.

[22] S. Chen, A. Livingstone, L. Hanzo, "Minimum bit-error rate design for space-time equalization-multiuser detection", IEEE Trans. Commun., vol. 54, no. 5, pp. 824-832, 2006. DOI: 10.1109/JPROC.2010.2042415. 